\title{
Five years' experience of combined intralesional therapy in infantile hemangioma
}

\author{
Ahmed Abdelghaffar Helal ${ }^{1,2^{*}}$ (D) and Mohammad Alsayed Daboos ${ }^{1}$
}

\begin{abstract}
Background: Infantile hemangiomas $(\mathrm{IH} s)$ are common vascular tumors. Although it involutes spontaneously, outcomes are unpredictable. Intralesional therapy is one of its treatment modality. We present our experience with combined intralesional therapy for $1 \mathrm{Hs}$ over a 5-year duration. A total of 427 patients were treated and followed at Al-Azhar University Hospitals during the study period of 5 years. All patients were treated by intralesional therapy in the form of combined injection of triamcinolone and bleomycin. All patients were followed for the response. Response to the treatment was graded as marked, partial, and poor improvement.

Results: IHs were noticed within the first month of life in $90.2 \%$ of patients. The commonest site of involvement was head and neck in $90 \%$ of patients. The commonest clinical presentation was swelling with discoloration. Mean age was $7.43 \pm 6.04$ months and mean IHs size was $15.54 \pm 11.13 \mathrm{~cm}^{2}$. The response to the treatment was highest for patients below 1 year of age. The reported complications were ulceration, scarring, and subcutaneous atrophy in some cases.

Conclusion: Combined intralesional therapy in IHs showed good efficacy in most patients. It is a reliable and safe treatment modality with clear curative effects and minimal complications. If IHs treatment is indicated, combined intralesional therapy should be considered as an alternative effective treatment modality.
\end{abstract}

Keywords: Infantile hemangioma, Combined intralesional therapy, Bleomycin, Corticosteroids

\section{Background}

Infantile hemangiomas (IHs) are the most common tumors of infancy, characterized by pathologic endothelial cell proliferation. Currently, there are multiple treatment modalities for IHs. In many cases, no treatment is required. However, prompt intervention may be strongly considered in up to $20 \%$ of cases. The most important rationale for intervention is the presence of life or function altering complications. There have been various studies suggesting beneficial effects of intralesional therapy of corticosteroids and bleomycin in IHs. However, studies evaluating the combined intralesional treatment of both corticosteroids with Bleomycin are lacking [1-5].

Herein, we present a prospective study of combined intralesional therapy in IHs. Combined intralesional

\footnotetext{
* Correspondence: helalhmada@yahoo.com

${ }^{1}$ Pediatric Surgery Department, Al-Azhar University, Cairo, Egypt

${ }^{2}$ Al-Houssain University Hospital, Darrasa, Cairo, Egypt
}

injection of corticosteroids and bleomycin was used. Special emphasis was given to indication for treatment, safety, efficacy, and any local or systemic adverse effects of treatment.

\section{Methods}

This prospective study included 427 patients with IHs, attending the hemangioma clinic at Al-Azhar University Hospitals between January 2013 and January 2019. The diagnosis was based on history and clinical examination and confirmed by Doppler ultrasonography for all cases. The criteria for study enrollment were complicated IHs (pain, bleeding, ulceration, or unaesthetic appearance), IHs causing functional impairments (periocular, ear, tongue, anogenital lesions), IHs with slow involution or scarring (parotid, lip lesions), patients who cannot tolerate propranolol treatment (children with cardiac abnormalities, parent refusal for propranolol treatment, or side effects of oral propranolol (severe bradycardia, 
severe hypoglycemia). Port wine hemangiomas, vascular tumors other than IHs, arteriovenous malformations, IHs with intracranial extension and all patients receiving previous systemic treatment for their IHs were excluded from our study to avoid misinterpretation of the results.

All IHs patients fulfilling the criteria for study enrolment were submitted to combined intralesional injection of triamcinolone (at a dose of $1 \mathrm{mg} / \mathrm{kg} /$ injection) and bleomycin, (at a dose of $0.2 \mathrm{mg} / \mathrm{kg} /$ injection).

\section{Patient management protocol}

\section{First visit}

For all patients, the following were recorded; age, sex, site, size of lesion (measured in 2 access perpendicular to each other using soft tape), numbers of lesions, time of appearance, cause of treatment decision, photography of the lesion and family history. Laboratory investigations include complete blood picture, prothrombin time, and activated partial thromboplastin time. Morning serum cortisol, adrenocorticotropic hormone (ACTH), and fasting serum glucose were measured, height, weight, and blood pressure were also recorded. Radiological investigations include; Doppler ultrasonography and abdominal ultrasonography.

\section{Injection technique}

Before injection, the lesion was divided roughly into four quadrants and each quadrant was injected at one time. To minimize bleeding, the lesions were injected using a 26-gauge needle entering through normal skin and advancing toward the lesion, in a radial pattern. After injection, local pressure was applied for $5 \mathrm{~min}$. Analgesia was prescribed for first $24 \mathrm{~h}$ then on demand. For parotid lesions the injection was done under ultrasound guidance. Patients with tongue hemangioma need general anesthesia. The interval between injections was 1 month, and number of injections was 7 injections at one therapeutic period (range 2-6).

\section{Follow-up visits (every 1 month)}

For all patients before subsequent injection, clinical examination, photographs, and Doppler ultrasonography were done to evaluate response to treatment. Height, weight, and blood pressure were recorded at each visit. Morning serum cortisol, adrenocorticotropic hormone (ACTH), fasting serum glucose were measured, to exclude corticosteroid side effects (morning serum cortisol concentration of less than $5 \mathrm{ng} / \mathrm{mL}$ was considered adrenal suppression).

The response to treatment was evaluated clinically by cessation of growth or decrease in size, lightening of lesion color, flattening of lesion surface, and Doppler ultrasonography to evaluate lesion blood flow (Figs. 1, 2, and 3). The clinical improvement outcomes were followed every month in the regular weekly Wednesday Grand Round Meeting and clinical improvement was evaluated by our 4 senior pediatric surgeons in pediatric surgery department at Al-Hussein University Hospital.

\section{Results}

The Patients' demographic data, lesions size, number of visits, and follow-up is shown in (Table 1). The total number of patients attending the hemangioma clinic was 427 , of which 87 were lost to continue the follow-up, and they were excluded from the results. So results were based on 340 patients. The overall male-to-female ratio was 1:4.

The commonest site of involvement was head and neck in 306 patients (90\%), abdomen and trunk in 20 patients $(5.8 \%)$, and anogenital region in 14 patients (4.1\%). In 306 patients (90\%), IH was noticed within the first month of life. In 34 patients (10\%), IH was noticed within the first 3 months of life. The clinical presentation was swelling with discoloration in all patients (100\%), cosmetic disfigurement in 272 patients (80\%), ulceration in 34 patients $(10 \%)$, functional disability in 17 patients (5\%), and bleeding in 17 patients (5\%).

The start of treatment was before 1 year of age in 289 patients (85\%) and after 1 year of age in 51 patients (15\%). The overall mean age was $7.43 \pm 6.04$ months. The mean numbers of injections were $5.28 \pm 0.50$ (range, 1-7). Patient younger than 1 year showed better improvement than children older than 1 year.

The overall mean IH size was $15.54 \pm 11.13 \mathrm{~cm}^{2}$ with marked decreasing of mean lesions size at the end of

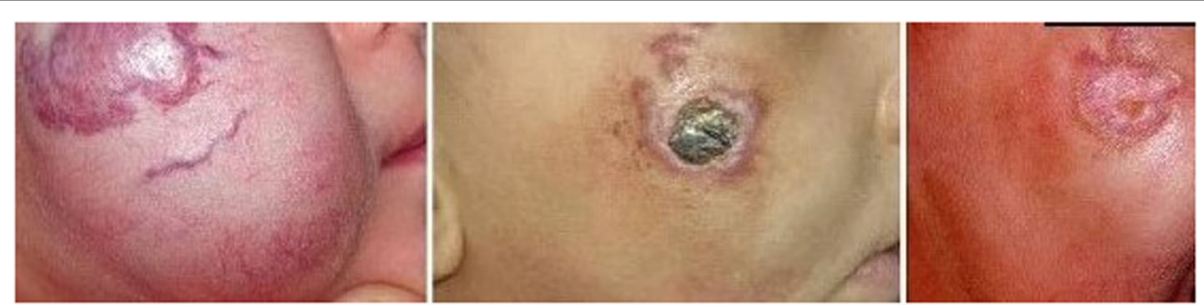

Fig. 1 Female child 5 months old with large cheek $\mathrm{IH}$ (with right visual axis obstruction). a $\mathbb{H}$ pretreatment. b After 6 injections showing marked regression in size (about 90\%) with healing ulcer in the center of lesion (note improvement in visual axis as the eye is well opened). c Healed ulcer 


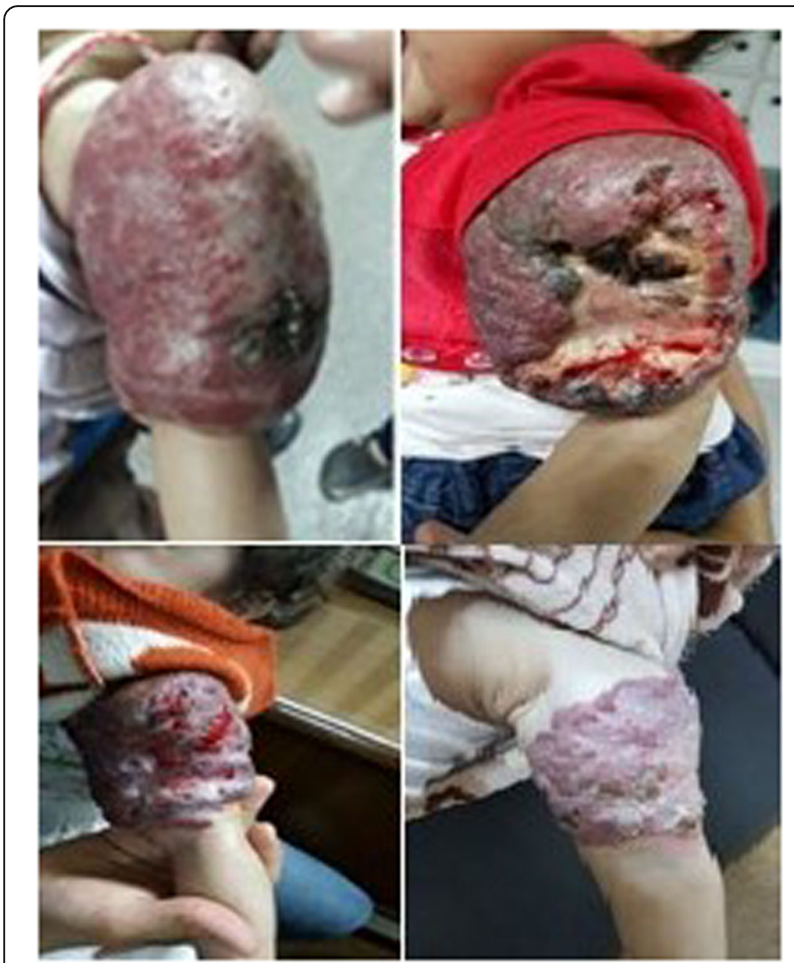

Fig. 2 Female patient with left shoulder before and after injection. Note the ulceration during the course of treatment, and the healed ulcer with marked involution of the lesion in the last picture

study about $(1.4 \mathrm{~cm} \pm 9.8)$ with highly significant improvement ( $P$ value .00156) (Table 2$)$

Marked improvement was reported in 300 cases (88\%), partial improvement in 20 cases (6\%), and poor improvement in 20 cases $(6 \%)$.

(Marked improvement equal 75 to $90 \%$ shrinkage of the lesion with marked decrease in lesion blood flow by Doppler ultrasound, partial improvement (50 to 75\% shrinkage of the lesion size) with moderate decrease in lesion blood flow, poor improvement less than 50\%
Table 1 Demographic data of patients

\begin{tabular}{ll}
\hline Demographic data & Pre therapy parameters \\
\hline Age $($ mean \pm SD) & $\begin{array}{l}7.43 \pm 6.04 \text { months } \\
\text { (range, 3-49 months) }\end{array}$ \\
Size of hemangioma (mean \pm SD) & $15.54 \pm 11.13 \mathrm{~cm}^{2}$ \\
& $\left(\right.$ range, $\left.0.5-19 \mathrm{~cm}^{2}\right)$ \\
No. of visits (mean \pm SD) & $7.83 \pm 2.18$ \\
& (range, 2-14) \\
\hline
\end{tabular}

shrinkage of the lesion size) with minor decrease in lesion blood flow.

The pretreatment serum ACTH and cortisol concentrations were normal in all patients. The follow-up serum $\mathrm{ACTH}$ and cortisol concentrations were normal in all patients (no adrenal suppression). Also, height and weight percentiles did not differ after therapy. None of the patients developed growth delay or cushioned appearance. Blood glucose and blood pressure were within normal ranges during therapy. The reported complications were ulceration in 30 patients $(8.8 \%)$, scarring in 10 patients (2.9\%) and subcutaneous atrophy in 7 patients (2\%). All complications are summarized in (Table 3). All patients were followed for 6 months' post-treatment.

\section{Discussion}

IHs are one of most common tumors of infancy. Although benign neglect is the cornerstone for its treatment, sometimes the final results are unpredictable and incomplete, moreover the time period for complete involution is not definite. This time period may cause severe parents anxiety; therefore, intervention is required [6-8].

In most studies, the intervention for IHs is required for lesions which may interfere with vital structure or function; this is true for lesions in airway, gastrointestinal tract, and lesions in the periorbital region, in addition to very large rapidly growing $\mathrm{IH}$, which was more liable to ulceration and/or bleeding, IHs with

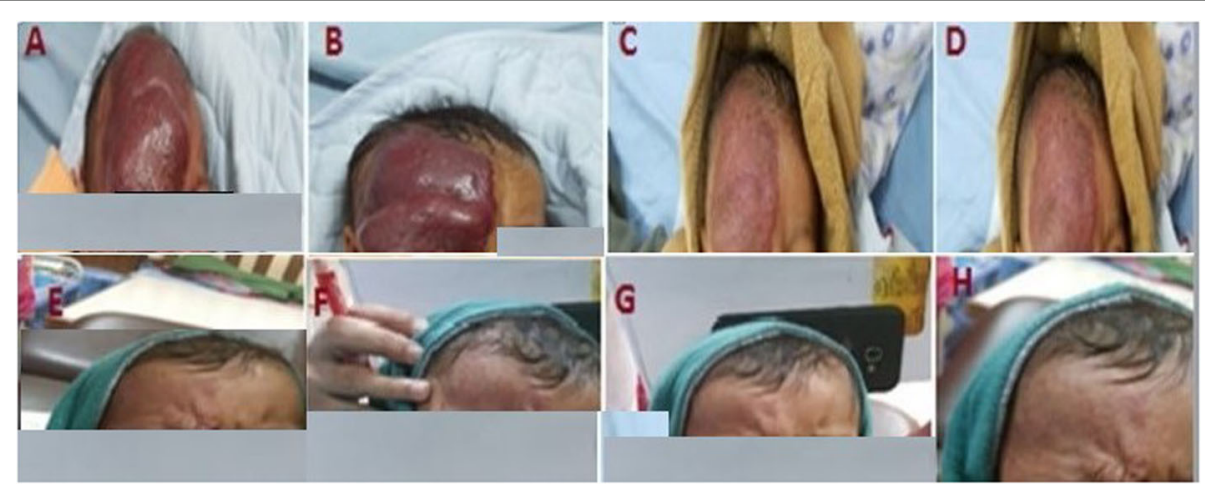

Fig. 3 Female patient with large forehead hemangioma extending to the right eye before and after injection. Showed marked improvement 
Table 2 Difference between mean lesion size pre and post therapy

\begin{tabular}{lll}
\hline $\begin{array}{l}\text { Mean Lesion size before } \\
\text { therapy }\end{array}$ & $\begin{array}{l}\text { Mean Lesion size after } \\
\text { therapy }\end{array}$ & $\begin{array}{l}P \\
\text { value }\end{array}$ \\
\hline $15.54 \pm 11.13$ & $1.4 \pm 9.8$ & .00156 \\
\hline
\end{tabular}

increased risk of scarring or disfigurement, as lesions of the nose, lip, ear, and large hemangiomas of the face. In the current study, the indications for intervention were required for patients with complicated $\mathrm{IH}$, pain, bleeding, ulceration, or unaesthetic appearance, IHs that occlude vital structures as ear (decreased auditory conduction) and tongue (impaired ventilation and feeding); IHs associated with slow involution or scarring as parotid lesions, lip lesions, lesions on the tip of the nose, or anogenital lesions [9-14].

The treatment modalities for IHs include systemic corticosteroids, non-selective B blocker (propranolol). However, the use of systemic corticosteroids has a potential side effect and the long duration of the treatment and is not widely used today. Propranolol has been shown to inhibit vascular proliferation of capillary hemangioma. Although it has been accepted as an effective treatment option for hemangiomas and considered as first-line treatment, it has serious reported complications in some children with systemic propranolol treatment as hypersomnolence, reflux, bronchospasm, sever hypotension, severe hypoglycemia, and hyperkalemia. In addition, parent compliance with the use of propranolol is mostly poor. Intralesional therapy for IHs is the second line of treatment in patients who are contraindicated or reported poor compliance with propranolol treatment. They showed good efficacy and high tolerability in children with IHs lesions [15-17]. There were various studies reporting beneficial effects of intralesional therapy of corticosteroids and bleomycin in IHs. However, studies evaluating the combined intralesional therapy using intralesional corticosteroids with bleomycin are limited. In the current study, combined intralesional injection of corticosteroids and bleomycin was used for treatment of IHs. Indication for treatment, safety, efficacy, and any local or systemic adverse effects of treatment were discussed.

Table 3 Complications of combined interalesional therapy

\begin{tabular}{ll}
\hline Complications & Percent \\
\hline Ulceration & 30 patients (8.8\%) \\
Subcutaneous atrophy & 7 patients (2\%) \\
Scarring & 10 patients (2.9\%) \\
Cushingoid facies & No \\
Hypertension & No \\
Growth retardation & No \\
\hline
\end{tabular}

The reported Cushingoid appearance after intralesional steroid injection ranged from 1 to $3 \%$. However, other studies did not show Cushingoid changes after intralesional steroid injection. Morkane et al. [17] reported adrenal suppression and failure to thrive following injection of infantile hemangiomas with high dose of triamcinolone $(4-10 \mathrm{mg} / \mathrm{kg}$ per injection). Emir et al. used intralesional steroid injection for treatment of $\mathrm{IHs}$ in six patients at a dose of $2 \mathrm{mg} / \mathrm{kg}$ for $2-5$ injections at monthly intervals and they reported that all patients in their study had adrenal suppression during treatment, and they concluded that the use of intralesional steroid therapy is not a superior treatment option for hemangiomas. It also has side effects comparable to systemic steroids. Couto and Greene published a study looking at efficacy and safety of intralesional triamcinolone in 100 infants. All tumors responded (63\% regressed and 37\% stabilized) and none of the patient had systemic absorption [18-20].

In the current study, the reported complications of treatment were ulceration in 30 patients $(8.8 \%)$ and scarring in 10 patients $(2.9 \%)$. Subcutaneous atrophy in $7 \mathrm{pa}-$ tients $(2 \%)$. the pretreatment serum ACTH and cortisol concentrations were normal in all patients. The followup serum ACTH and cortisol concentrations were normal in all patients during treatment (no adrenal suppression). Also, height and weight percentiles did not differ during therapy. None of the patients developed growth delay or Cushingoid appearance. Blood glucose and blood pressure were within normal ranges during therapy. We may explain these results with the use of very small dose of corticosteroid ( $1 \mathrm{mg} / \mathrm{kg}$ per injection), supporting what is reported in the literature that these complications increased with the use of larger doses and long duration of corticosteroid therapy [21-23].

The initial report with intralesional Bleomycin injection by Kullendorff in 1997 includes five children, with painful massive, inoperable IHs. All his treated children were relieved of pain, and the swelling was reduced with no complications or side effects. Muir et al. evaluated the effectiveness of intralesional Bleomycin injection in 37 patients. They reported complete resolution or significant improvement in $87 \%$ of the patients. In another study undertaken by Omidvari et al. assessed the effectiveness of intralesional bleomycin injection with 2 weeks intervals for 4 to 6 times in treatment of 32 patients with complicated IHs. They observed that the maximum of lesion regression occurred in the first three months of treatment. They had a follow-up of 6 months and reported no recurrence or systemic and local complications. They concluded that bleomycin is a good therapeutic modality for treatment of IHs especially in painful or massive lesions. Hassan et al. treated 75 patients with intralesional bleomycin injection and found 
complete resolution in (24\%) patients, marked improvement occurred in $(47 \%)$ patients, mild improvement in (18.5\%) patients, and no cure in (10.5\%) cases [24-28].

The reported reactions that commonly occur immediately after intralesional bleomycin injection include local erythema, swelling, and pain. Bleeding, ulceration, cellulitis, hypopigmentation, transient alopecia, and flu-like symptoms may also occur, lymphangitis, flagellate hyperpigmentation, and rarely Raynaud's phenomenon. Pulmonary fibrosis is the most important dose-dependent complication of systemic bleomycin therapy but has not been reported after intralesional bleomycin injection, as the dosage used in sclerotherapy is much lower than those used in oncology. Chaudry et al. performed pulmonary function tests and radiographs on 31 patients and did not observe pulmonary abnormalities in a mean follow-up of 3.2 years [29-34]. In the current study, the observed complications of treatment were ulceration in 30 patients $(8.8 \%)$, scarring in 10 patients $(2.9 \%)$, and subcutaneous atrophy in 7 patients $(2 \%)$. Beside some local complications in the form of local erythema, swelling, and pain which were self-limiting and managed with analgesia and topical sousing creams.

Yang et al. used intralesional bleomycin plus dexamethasone for treatment of 31 children with infantile parotid hemangiomas. They reported that the addition of corticosteroids, with its known anti-allergic and antiinflammatory properties, may inhibit a possible allergic reaction from bleomycin. Also, it may reduce the excessive formation of scar. Moreover, corticosteroids by itself play a role in controlling growth and possible regression of IHs. They used the term controlling therapy, because they advocate intervention in the early and proliferative phase of IHs, with small dosage and low concentration of both drugs to inhibit the rapid $\mathrm{IH}$ growth. In the meantime, they try to keep the space for natural involution of the lesions to occur. They concluded that the role of both drugs interaction and natural IH involution should be considered and interpretation about the effects of intralesional bleomycin injection with addition of corticosteroids was difficult to make solid conclusion that the improvement seen was from the bleomycin and not the corticosteroids or that the reverse is true $[35,36]$..

In the current study, from the authors point of view, bleomycin plays a leading role in the regression of IHs growth and that the synergetic effect of corticosteroids is responsible for the rapid regression of the lesions observed in the patients treated with this combined intralesional therapy. Also the interval between injections was important, this interval was determined according to stage of treatment, the lesion's size, blood flow (by Doppler ultrasound), and treatment response. In the earlier stage of treatment and for large-size lesions with abundant blood flow, we used a relatively short interval between injections ( 3 weeks) for initial growth control. On the other hand, in the later stages of treatment, this interval was extended (from 1.5 to 2 month) to allow more time for observation of the tumor response to treatment after initial growth control and in the meantime to keep the space for natural involution of the lesions to occur. The injection was stopped if the lesion's size reduced more than $50 \%$, when abundant blood flow could not be detected by Doppler or after 7 injections. Also, it was observed that improvement was better in patients younger than 1 year and this may be due to the intervention during the proliferative phase of the hemangioma cycle.

\section{Conclusion}

Combined intralesional therapy in IHs showed good efficacy in most patients. It is reliable and safe treatment modality with clear curative effects and minimal complications. If IHs treatment is indicated, combined intralesional therapy should be considered as an alternative effective treatment modality.

\section{Abbreviations}

IHs: Infantile hemangiomas

\section{Acknowledgment \\ The authors provide great acknowledgment to Wednesday Grand Round Professors, Pediatric Surgery Department, Al-Hussein University Hospital for their great help and kind care follow-up of patients.}

Ethical approval and consent to participate

The study was approved by our Hospital's Ethics Committee (number 00004), and written informed consent was obtained from parents.

\section{Authors' contributions}

AH performed the intralesional injection treatment and follow-up of patients and was a major contributor in writing the manuscript. MA analyzed and interpreted the patient data and performed the statistical analysis. All authors have read and approved the final manuscript.

\section{Funding \\ None}

Availability of data and materials

Not applicable for that section.

\section{Competing interest}

The authors declare that they have no competing interests.

\section{Consent for publication}

Written informed consent for publication was obtained from parents of all study participants and approved by our Hospital's Ethics Committee.

Received: 2 October 2019 Accepted: 6 November 2019

Published online: 12 December 2019

\section{References}

1. Ahuja T, Jaggi N, Kalra A, Bansal K, Sharma SP. Hemangioma: review of literature. The journal of contemporary dental practice. 2013;14(5):1000-7.

2. Antonescu C. Malignant vascular tumors--an update, Modern pathology: an official journal of the United States and Canadian Academy of Pathology. Inc. 2014;27(Suppl 1):S30-8. 
3. Arkan Hadi A, Ashraf Hashim A, Fadel Abbas A. Intralesional triamcinolone injection in the management of cutaneous hemangiomas in children. Medical Journal of Babylon. 2012;9(1):182-7.

4. Berlien HP. Classification of infantile hemangiomas and other congenital vascular tumors. In: Mattassi R, Loose DA, Vaghi M, editors. hemangiomas and vascular malformations: an atlas of diagnosis and treatment. Milano: Springer Milan; 2009. p. 23-34.

5. Boscolo E, Bischoff J. Vasculogenesis in infantile hemangioma. Angiogenesis 2009;12(2):197-207.

6. Bota M, Popa G, Blag C, Tataru A. Infantile hemangioma: a brief review. Clujul medical. 2015;88(1):23-7.

7. Buckmiller LM. Update on hemangiomas and vascular malformations. Current opinion in otolaryngology \& head and neck surgery. 2004;12(6):476-87.

8. Buckmiller LM, Francis CL, Glade RS. Intralesional steroid injection for proliferative parotid hemangiomas. International journal of pediatric otorhinolaryngology. 2008;72(1):81-7.

9. Buckmiller LM, Munson PD, Dyamenahalli U, Dai Y, Richter GT. Propranolol for infantile hemangiomas: early experience at a tertiary vascular anomalies center. The Laryngoscope. 2010;120(4):676-81.

10. Chambers CB, Katowitz WR, Katowitz JA, et al. A controlled study of topical $0.25 \%$ timolol maleate gel for the treatment of cutaneous infantile capillary hemangiomas. Ophthal Plast Reconstr Surg. 2012;28:103-6.

11. Chan SA, Goodyear HM. Infantile haemangioma: their presentation and management. British journal of hospital medicine. 2015;76(9):523-7.

12. Chang, L. C., et al. (2008), Growth characteristics of infantile hemangiomas:

13. Chantharatanapiboon W. Intralesional corticosteroid therapy in hemangiomas: clinical outcome in 160 cases. Journal of the Medical Association of Thailand. 2008;91(Suppl 3):S90-6.

14. Chaudry G, Guevara CJ, Rialon KL, Kerr C, et al. Safety and efficacy of bleomycin sclerotherapy for microcystic lymphatic malformation. Cardiovascular and interventional radiology. 2014;37(6):1476-81.

15. Chen MT, Yeong EK, Horng SY. Intralesional corticosteroid therapy in proliferating head and neck hemangiomas: a review of 155 cases. Journal of pediatric surgery. 2000;35(3):420-3.

16. Chen TS, Eichenfield LF, Friedlander SF. Infantile hemangiomas: an update on pathogenesis and therapy. Pediatrics. 2013:131(1):99-108.

17. Morkane C, Gregory JW, Watts P, Warner JT. Adrenal suppression following intralesional corticosteroids for periocular haemangiomas. Archives of disease in childhood. 2011;96(6):587-9.

18. Emir S, Gurlek Gokcebay D, Demirel F, Tunc B. Efficacy and safety of intralesional corticosteroid application for hemangiomas. Turkish journal of medical sciences. 2015;45(2):335-8.

19. Couto JA, Greene AK. Management of problematic infantile hemangioma using intralesional triamcinolone: efficacy and safety in 100 infants. Journal of plastic, reconstructive \& aesthetic surgery: JPRAS. 2014;67(11):1469-74.

20. Chinnadurai S, Fonnesbeck C, Snyder KM, Sathe NA, et al. Pharmacologic interventions for infantile hemangioma: a meta-analysis. Pediatrics. 2016; 137(2):e20153896.

21. Cioffi A, Reichert S, Antonescu CR, Maki RG. Angiosarcomas and other sarcomas of endothelial origin. Hematology/oncology clinics of North America. 2013;27(5):975-88.

22. Clarke, L. (2009), Studies on the mechanism of action of the chemotherapeutic drug bleomycin on cell lines derived from haemangioma and keloid, Masters thesis, Durham University.

23. Kullendorff CM. Efficacy of bleomycin treatment for symptomatic hemangiomas in children. Pediatric surgery international. 1997;12(7):526-8.

24. Muir T, Kirsten M, Fourie P, Dippenaar N, Ionescu GO. Intralesional bleomycin injection ( $\mathrm{BI} \mid$ ) treatment for haemangiomas and congenital vascular malformations. Pediatric surgery international. 2004;19(12):766-73.

25. Omidvari S, Nezakatgoo N, Ahmadloo N, Mohammadianpanah M, Mosalaei A. Role of intralesional bleomycin in the treatment of complicated hemangiomas: prospective clinical study. Dermatologic Surgery. 2005;31(5):499-501.

26. Hassan Y, Osman AK, Altyeb A. Noninvasive management of hemangioma and vascular malformation using intralesional bleomycin injection. Annals of plastic surgery. 2013;70(1):70-3.

27. Masiha $H$, Hasani ME, Emami AH, Jafari M, Manafi $A$. The synergistic effect of bleomycin, triamcinolone and epinephrine in treatment of hemangioma and arteriovenous malformations. World J Plast Surg. 2012;1(2):83-90.

28. Kagami S, Kuwano Y, Shibata S, et al. Propranolol is more effective than pulsed dye laser and cryosurgery for infantile hemangiomas. Eur. J. Pediatr. 2013;172:1521-6.
29. M. Luu and I.J. Frieden (2013): Haemangioma: clinical course, complications and management. British Journal of Dermatology 169, pp20-30.

30. Sheilagh M. Maguiness, MD, and Ilona J. Frieden, (2010): Current management of infantile hemangiomas, Semin Cutan Med Surg 29:106-114.

31. Vasani RJ, Khanna D, Singal A. Cutaneous vascular lesions and their management in Indian setting. Dermatologic therapy. 2012;25(4):358-75.

32. Vlachakis I, Gardikis S, Michailoudi E, Charissis G. Treatment of hemangiomas in children using a Nd:YAG laser in conjunction with ice cooling of the epidermis: techniques and results. BMC pediatrics. 2003;3:1-6.

33. Yang $Y$, Sun $M$, DDS, Cheng $X$, et al, (2009): Bleomycin A5 plus dexamethasone for control of growth in infantile parotid hemangiomas, oral medicine Vol. 108 No. 1 July 62-69.

34. Wang XK, Zhao YF. A practical guide to treatment of infantile hemangiomas of the head and neck. International journal of clinical and experimental medicine. 2013:6(10):851-60

35. Xu SQ, Jia RB, Zhang W, Zhu H, Ge SF, Fan XQ. Beta-blockers versus corticosteroids in the treatment of infantile hemangioma: an evidencebased systematic review. World journal of pediatrics: WJP. 2013;9(3):221-9.

36. Zheng JW, Zhang L, Zhou Q, Mai HM, Wang YA, Fan XD, Qin ZP, et al. Propranolol therapy for infantile haemangiomas: review of the literature. International journal of pediatric otorhinolaryngology. 2010;74(4):338-42.

\section{Publisher's Note}

Springer Nature remains neutral with regard to jurisdictional claims in published maps and institutional affiliations.

\section{Submit your manuscript to a SpringerOpen ${ }^{\circ}$ journal and benefit from:}

- Convenient online submission

- Rigorous peer review

- Open access: articles freely available online

High visibility within the field

- Retaining the copyright to your article

Submit your next manuscript at $>$ springeropen.com 